\title{
Monoclonal Antibody NEO-201
}

National Cancer Institute

\section{Source}

National Cancer Institute. Monoclonal Antibody NEO-201. NCI Thesaurus. Code C156398.

A humanized immunoglobulin G1 (IgG1) monoclonal antibody derived from an immunogenic preparation of tumor-associated antigens (TAAs) from pooled allogeneic colon cancer tissue extracts, with potential antineoplastic and immunomodulatory activities. Upon intravenous administration, monoclonal antibody NEO-201 targets and binds to malignant tissues with tumor-specific mutations in the membrane-anchored proteins, carcinoembryonic antigen-related cell adhesion molecules 5 and 6 (CEACAM5 and CEACAM6). This prevents the interaction between tumor cell CEACAM 5 and natural killer (NK) cell CEACAM1, and reverses CEACAM1-dependent inhibition of NK cytotoxicity. This may result in the activation of NKs and results in NK-mediated tumor cell killing. Additionally, monoclonal antibody NEO-201 may activate innate immune responses against tumor cells such as antibody-dependent cellular cytotoxicity (ADCC) and complement-dependent cytotoxicity (CDC). CEACAM 5, and 6 are members of the CEA family of proteins. These membrane proteins are over expressed in a variety of cancer cell types and play a key role in cell migration, invasion, and adhesion. 